Review

\title{
Treatment implications of natural compounds targeting lipid metabolism in nonalcoholic fatty liver disease, obesity and cancer
}

\author{
Can Cheng ${ }^{1,2,3^{*}}$, Songming Zhuo ${ }^{*}$, Bo Zhang 5 , Xu Zhao 1,2,3, Ying Liu' ${ }^{6}$, Chaoliang Liao ${ }^{1,2,3}$, Jing Quan $^{1,2,3}$, \\ Zhenzhen Li ${ }^{1,2,3}$, Ann M. Bode ${ }^{7}$, Ya Cao ${ }^{1,2,3,8}$, Xiangjian Luo ${ }^{1,2,3,8,}, \mathbb{}$
}

1. Key Laboratory of Carcinogenesis and Invasion, Chinese Ministry of Education, Xiangya Hospital, Central South University, Changsha, Hunan 410078, PR China.

2. Cancer Research Institute and School of Basic Medical Science, Central South University, Changsha, Hunan 410078, PR China.

3. Key Laboratory of Carcinogenesis, Chinese Ministry of Health, Changsha, Hunan 410078,PR China.

4. Department of Respiratory Medicine, Shenzhen Longgang Center Hospital, Shenzhen, Guangdong 518116, PR China.

5. Department of Ultrasound Imaging, Xiangya Hospital, Central South University, Changsha, Hunan 410078, PR China.

6. Department of Medicine, Hunan Traditional Chinese Medical College, Zhuzhou, Hunan 412000, China.

7. The Hormel Institute, University of Minnesota, Austin, MN 55912, USA.

8. Molecular Imaging Research Center of Central South University, Changsha, Hunan 410078, China.

* These authors contribute equally to the paper

$\square$ Corresponding author: Xiangiian Luo

(c) Ivyspring International Publisher. This is an open access article distributed under the terms of the Creative Commons Attribution (CC BY-NC) license (https://creativecommons.org/licenses/by-nc/4.0/). See http://ivyspring.com/terms for full terms and conditions.

Received: 2019.02.04; Accepted: 2019.05.19; Published: 2019.06.04

\begin{abstract}
Metabolic disorders can lead to a scarcity or excess of certain metabolites such as glucose, lipids, proteins, purines, and metal ions, which provide the biochemical foundation and directly contribute to the etiology of metabolic diseases. Nonalcoholic fatty liver disease, obesity, and cancer are common metabolic disorders closely associated with abnormal lipid metabolism. In this review, we first describe the regulatory machinery of lipid metabolism and its deregulation in metabolic diseases. Next, we enumerate and integrate the mechanism of action of some natural compounds, including terpenoids and flavonoids, to ameliorate the development of metabolic diseases by targeting lipid metabolism. Medicinal natural products have an established history of use in health care and therapy. Natural compounds might provide a good source of potential therapeutic agents for treating or preventing metabolic diseases with lipid metabolic abnormalities.
\end{abstract}

Key words: natural compound; lipid metabolism; nonalcoholic fatty liver disease; obesity; cancer

\section{Deregulation of lipid metabolism in metabolic diseases}

\subsection{Lipid metabolism}

Lipids comprise triglycerides (TAGs) and lipoids (e.g., phospholipids and sterols) and TAGs are the major forms of energy storage. Lipids provide energy and essential fatty acids (FAs) for the human body and are also essential components of cells and tissues, such as plasma membrane and myelin.

Here we focus on FA metabolism. The first step in FA synthesis is the carboxylation of acetyl-CoA to malonyl-CoA, which is catalyzed by acetyl-CoA carboxylase (ACC), a rate-limiting enzyme for FA biosynthesis[1]. Acetyl-CoA and malonyl-CoA synthesize saturated FAs, which are catalyzed by the multifunctional enzyme, fatty-acid synthase (FASN). Desaturation of FAs is catalyzed by stearoyl-CoA desaturases(SCDs), which produce mono-unsaturated FAs used for the synthesis of phosphoglycerides and bioenergy stored in the form of TAGs[2].

In the process of FAs degradation, TAGs are broken down into free FAs and glycerol and this step is catalyzed by lipases such as monoacylglycerol 
lipase (MAGL), triglyceride lipase (ATGL) and hormone-sensitive lipase (HSL), which is the rate-limiting enzyme of lipolysis[3]. Acyl-CoA synthetase catalyzes the activation of FAs to produce acyl-CoAs, which are unable to move across the inner mitochondrial membrane [4]. Thus, the carnitine shuttle system is required for acyl-CoA entry into the mitochondrial matrix to be utilized for long-chain FA oxidation. Carnitine palmitoyl transferase-1 (CPT-1) transports long-chain FAs to mitochondria and is considered to be a rate-limiting enzyme for FA $\beta$-oxidation (FAO). Under the catalysis of enzymes, such as fatty acyl-CoA dehydrogenase and enoyl hydratase in the mitochondrial matrix, FAs are repeatedly cleaved to generate acetyl-CoAs, the process of which is referred to as FAO. Acetyl-CoAs are further fed into the tricarboxylic acid (TCA) cycle and coupled with oxidative phosphorylation to produce ATP [5].

\subsection{The regulation of lipid metabolism}

Under metabolic pressure such as hypoxia or nutrition deprivation, adenosine monophosphate-activated protein kinase (AMPK) is activated. AMPK, a heterotrimeric complex, consists of a catalytic subunit alpha(a), a regulatory subunit beta $(\beta)$ and a noncatalytic subunit gamma $(\gamma)$ [6]. AMPK can be phosphorylated and activated in the a-subunit at Thr172 by upstream kinases, including liver kinase B1 (LKB1), $\mathrm{Ca}^{2+} /$ calmodulin-dependent protein kinase kinase- $\beta$ (CaMKK), TGF-beta-activated kinase 1 (TAK1), or mixed-lineage kinase 3 (MLK3)[7]. AMPK maintains energy balance by decreasing ATP consumption and increasing ATP generation. It catalyzes the phosphorylation and inactivation of ACC and upregulates peroxisome proliferator-activated receptor a (PPARa), eventually stimulating FAO. Simultaneously, AMPK phosphorylates and activates malonyl-CoA decarboxylase (MCD), resulting in decreased malonyl-CoA, followed by reduced FA synthesis and release of CPT-1 inhibition. In addition, AMPK down-regulates sterol regulatory element binding protein 1c (SREBP-1c), the target genes which are FASN and SCD1. Therefore, AMPK plays important roles in the control of lipid metabolic homeostasis by stimulating FAO and inhibiting FA biosynthesis.

Several transcription factors are involved in adipogenesis and lipogenesis, including cAMP response element binding protein (CREB), CCAAT/enhancer binding protein (C/EBP) and peroxisome proliferator-activated receptors (PPARs). The PPAR subfamily contains three isoforms, PPARa, PPAR $\beta / \delta$ and PPAR $\gamma$. PPARa is a ligand-activated nuclear receptor and mediates peroxisomal and mitochondrial $\beta$-oxidation[8]. PPARa directly regulates the expression of medium-chain acyl-CoA dehydrogenase (MCAD), long-chain acyl-CoA dehydrogenase (LCAD) and very long-chain acyl-CoA dehydrogenase (VLCAD) to control the dehydrogenation of acyl-CoA. Furthermore, PPARa also promotes hepatic lipogenesis through its binding to the SREBP-1C promoter and enhancing its transcriptional activity in human primary hepatocytes. In mouse liver, PPARa agonists positively regulate SREBP-1c target genes, including FASN, ACC1 and SCD-1. Activation of PPAR $\beta / \delta$ in vivo has been demonstrated to increase lipid catabolism in skeletal muscle, heart and adipose tissues [9]. In addition, PPARY plays a substantial role in regulating lipid synthesis and differentiation of adipocytes and sebocytes[10].

\subsection{Abnormal lipid metabolism in metabolic diseases}

Liver, adipose tissue, and small intestine are the main tissues for the synthesis of TAGs, among which, liver, the major tissue for FAO and ketone body formation, plays a particularly important role in lipid metabolism. After synthesis in hepatic endoplasmic reticulum, TAGs are transported to extra-hepatic tissues in the form of very low-density lipoproteins (VLDL). The etiology of fatty liver is due to excessive accumulation of fat in liver cells caused by various factors, such as malnutrition or lack of essential FAs, choline, or protein. Lipid metabolism is involved in the development of nonalcoholic fatty liver disease (NAFLD) that has potential to progress to steatohepatitis, fibrosis, cirrhosis and hepatocellular carcinoma[11]. Disorders of lipogenesis and lipolysis can cause the accumulation of triglycerides in hepatocytes, which contributes to the progression of NAFLD[12, 13]. However, the pathogenesis of NAFLD remains to be elucidated and no established therapeutic approach against NAFLD is currently available.

Obesity is a chronic metabolic disease characterized by excessive fat accumulation in adipose tissue. Current evidence supports an intimate relationship between obesity and the development of NAFLD and obesity is one of the largest contributors to the development of this disease. Obesity is closely associated with a higher incidence of type 2 diabetes, cardiovascular disease and hepatic steatosis[14].

Cancer could be regarded as a systematic dysfunction of metabolic processes. Recently, increasing research findings reveal that lipid metabolism is substantially activated during carcinogenesis and malignant tumor progression [15-19]. In cancer cells, de novo FA synthesis is 
enhanced to provide for membrane formation, energy storage, and the generation of signaling molecules. FA oxidation is aberrantly activated in many types of tumors, including breast and colon cancers, specifically under conditions of glucose and oxygen deprivation.

Given that lipid metabolic dysfunction promotes the development of metabolic diseases, targeting lipid metabolism could be a promising strategy for prevention and therapy of these diseases [20].

\section{Natural compounds targeting lipid metabolism}

Most natural compounds are extracted from fungi and biological and marine organisms, and play important roles in defensive mechanisms against stress and pathogen attack. Natural compounds are an important source of innovative drugs. The world-famous chemical drugs derived from natural sources include aspirin, morphine, artemisinin, berberine and paclitaxel[21-25]. These drugs have made tremendous contributions to human health.
Here, we summarize the natural compounds reported to be involved in regulating lipid metabolism. Furthermore, we classify the compounds on the basis of their chemical structures and elucidate the mechanisms of each class of natural compounds in ameliorating metabolic diseases through their impact on lipid metabolism. Because of their novel chemical structures, diverse biological activities, and unique mechanisms of action, natural compounds have become an important source of drugs that can target metabolic diseases (Figure 1).

\subsection{Terpenoids and lipid metabolism}

Terpenoids are a common class of compounds found in plants. Terpenoids can be divided into monoterpenes, sesquiterpenes, diterpenes, sesterterpenes, triterpenes, tetraterpenes and polyterpenes based on the number of isoprene units. Emerging evidence suggests essential roles for terpenoids in alleviating metabolic diseases through their targeting of lipid metabolism (Table 1).

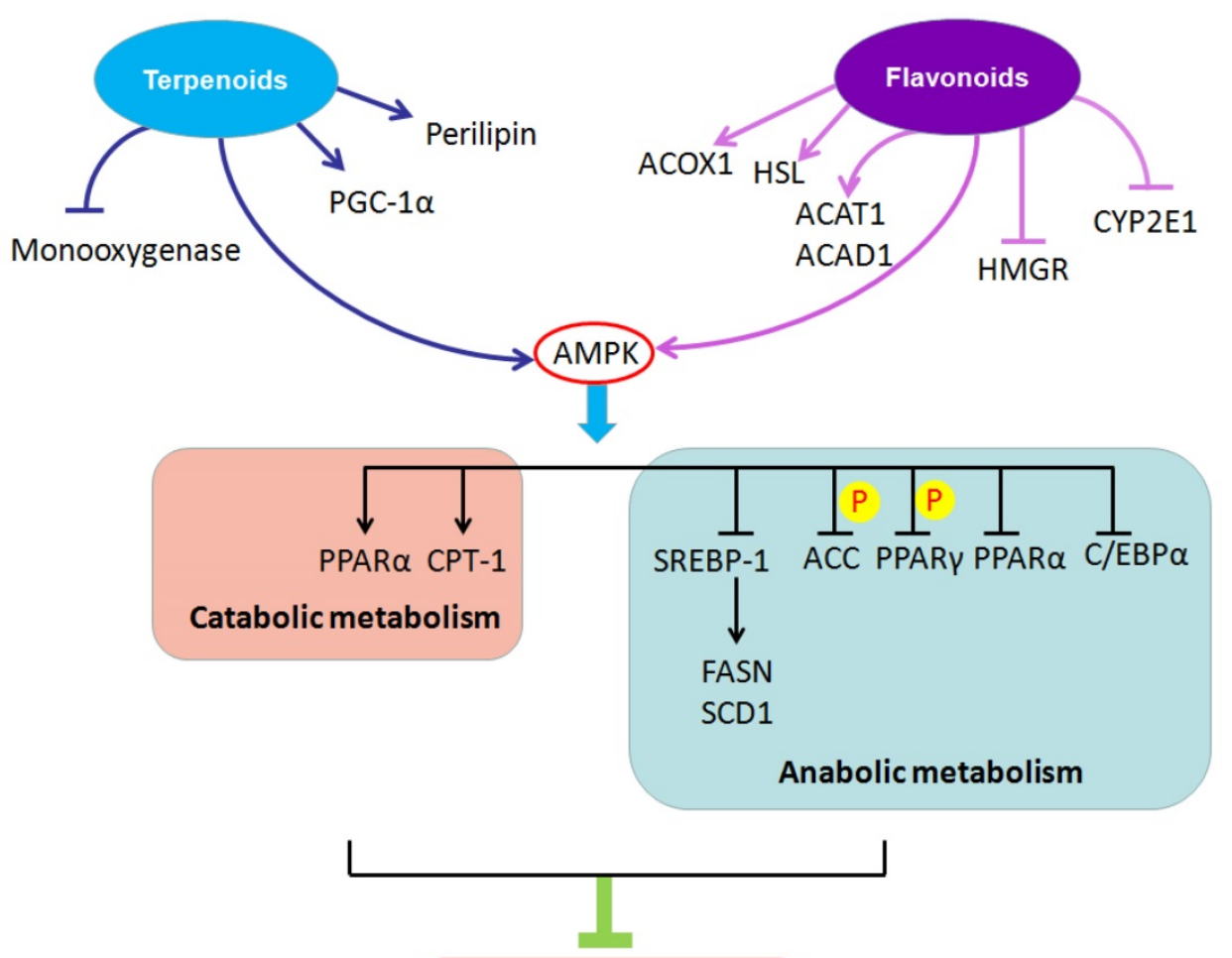

NAFLD, obesity and

cancer

Figure 1. Schematic of molecular mechanisms of natural compounds targeting lipid metabolism for the treatment of metabolic diseases. ACAT1, acetyl-CoA acetyltransferase 1; ACADI, acyl-CoA dehydrogenase 1; ACC, acetyl-CoA carboxylase; ACOX1, peroxisomal acyl-coenzyme A oxidase 1; AMPK, adenosine monophosphate- activated protein kinase; C/EBPa, CCAAT/enhancer binding protein a; CPT-1, carnitine palmitoyltransferase-1; CYP2E1, cytochrome P450 2E1; FASN, fatty-acid synthase; HMGR, 3-hydroxy-3-methylglutaryl-CoA reductase; HSL, hormone-sensitive lipase; PGC-1 $\alpha$, peroxisome proliferator-activated receptor Y coactivator-1 alpha; PPARa, peroxisome proliferator-activated receptor a; PPAR $\gamma$, peroxisome proliferator- activated receptor $\mathrm{Y}$; SCD1, stearoyl-coenzyme A desaturase 1; SREBP-1, sterol regulatory element binding protein 1. 
Table 1. Structure of terpenoids and their mechanisms of action targeting lipid metabolism

\begin{tabular}{|c|c|c|c|c|}
\hline Terpenoids & Structure & Effect on lipid metabolism & Research models & References \\
\hline \multirow[t]{3}{*}{ Betulinic acid } & & SCD-1 $\downarrow$ & in vitro & Potze L et al. 2016 [24] \\
\hline & & SREBP1 $\downarrow$ & in vitro & Quan HY et al. 2013 [28] \\
\hline & & $\mathrm{AMPK} \uparrow$ & in vitro & Quan HY et al. 2013 [28] \\
\hline \multirow[t]{3}{*}{ Ursolic acid } & & AMPK $\uparrow$ & in vivo & Lodi, A., et al.2017 [34] \\
\hline & & PPARa $\uparrow$ & in vitro & Jia Y et al. 2011 [32] \\
\hline & & LXR-SREBP1c pathway $\downarrow$ & in vitro & Jia Y et al. 2011 [32] \\
\hline \multirow[t]{2}{*}{ Andrographolide } & & SREBP, FASN, SCD $\downarrow$ & in vitro & $\begin{array}{l}\text { Chen CC et al. } 2016 \text { [38]; } \\
\text { Chen X et al. } 2017 \text { [41] }\end{array}$ \\
\hline & & CREB, C/EBP $\beta$, PPAR $\gamma \downarrow$ & in vivo & Jin L et al. 2012 [39] \\
\hline \multirow[t]{2}{*}{ Shizukaol D } & & $\mathrm{AMPK} \uparrow$ & in vitro & Hu R et al. 2013 [42] \\
\hline & & PPARa, PGC-1a $\uparrow$ & in vitro & $\begin{array}{l}\text { Vega RB, Huss JM and } \\
\text { Kelly DP } 2000[44]\end{array}$ \\
\hline \multirow[t]{2}{*}{ Ginsenoside Rb1 } & & AMPK, perilipin $\uparrow$ & $\begin{array}{l}\text { in vitro } \\
\text { in vivo }\end{array}$ & Yu X et al. 2015 [48] \\
\hline & & Cytochrome P-450 monooxygenase $\downarrow$ & in vivo & $\begin{array}{l}\text { Jenner AM and Timbrell } \\
\text { JA } 1994 \text { [49] }\end{array}$ \\
\hline
\end{tabular}

AMPK, adenosine monophosphate- activated protein kinase; FASN, fatty-acid synthase; LXRa, liver X receptor $a$; PGC-1a, peroxisome proliferator-activated receptor $\gamma$ coactivator-1 alpha; PPARa, peroxisome proliferator-activated receptor a; SCD1, stearoyl-coenzyme A desaturase 1; SREBP-1, sterol regulatory element binding protein 1.

\subsubsection{Betulinic acid (BetA)}

BetA is a plant-derived pentacyclic lupane triterpene. It induces apoptosis in multiple tumor types by a not fully understood, but in a mitochondria-dependent manner. Recently, BetA was reported to inhibit the activity of SCD-1, resulting in enhanced saturation levels of a mitochondrial lipid, cardiolipin (CL). In that CL impacts mitochondrial ultrastructure as well as function, the alteration of CL saturation leads to changes in mitochondrial morphology, release of cytochrome c, and induction of cell death [24].

Lipoprotein lipase plays an important role in the metabolism of lipoproteins and contributes to cellular lipid accumulation [26]. BetA attenuates the expression of macrophage-derived lipoprotein lipase and decreases cellular lipid accumulation, possibly through the inhibition of the protein kinase $C$ $(\mathrm{PKC}) /$ extracellular signal regulated kinases (ERKs)/c-Fos pathway.

SREBP1 mediates synthesis of FAs, phospholipids and triacylglycerols. LKB1 and CaMKK are two distinct upstream kinases that activate AMPK through phosphorylation on Thr172
[27]. Quan et al reported that BetA inhibits excessive triglyceride accumulation in HepG2 cells [28]. It also decreases SREBP1 activity, activates CaMKK, and up-regulates AMPK activity by phosphorylation, which results in reduced lipogenesis and lipid accumulation. BetA reduces hepatic steatosis by modulating the CaMKK/AMPK/SREBP1 signaling pathway and might be used to alleviate NAFLD [28].

BetA also exhibits inhibitory actions against carcinogenesis and metastatic progression. Its anticancer proprieties have been demonstrated against breast, prostate, stomach, pancreatic, ovarian and cervical carcinomas. BetA promotes apoptosis by down-regulating phosphatidylinositol 3-kinase (PI3K)/Akt signaling in human cervical cancer cells[23]. It induces cell death in part through its inhibition of SCD-1, which is frequently overexpressed in various tumor cells [24]. Overall, BetA exerts its effects on attenuating metabolic disease by directly or indirectly modulating the critical nodes of several lipid metabolic circuits, including SCD-1, SREBP1 and AMPK.

\subsubsection{Ursolic acid (UA)}

UA, a natural pentacyclic triterpenoid 
compound, is extracted from loquat leaves. It is also known as micromerolor malol and has the ability to mediate glucose and lipid metabolism [29, 30]. UA appears to stimulate lipolysis through the cAMP-dependent PKA pathway. It up-regulates adipose ATGL and promotes the translocation of HSL from the cytosol to lipid droplets. HSL is the rate-limiting enzyme for lipolysis and also catalyzes TAG hydrolysis [31]. UA increases the levels of PPARa, leading to enhancement of FAO and reduced lipogenesis by suppressing the liver $X$ receptor (LXR)-SREBP1c pathway. UA lowers intracellular triglyceride and cholesterol levels in hepatocytes, likely by inhibiting FA synthesis and enhancing FA uptake and oxidation [32].

UA might also be used as an alternative medicine for the treatment and prevention of cancer. In human T24 bladder cancer cells, activation of AMPK by UA contributes to inhibition of growth and increased apoptosis[33]. In prostate cancer, the combination of UA with resveratrol increases AMPK phosphorylation and decreases the activity of mammalian target of rapamycin complex 1 (mTORC1) [34]. Therefore, up-regulation of AMPK and PPARa or down-regulation of LXR-SREBP1c signaling to facilitate lipolysis mainly contributes to the effects of UA on alleviating metabolic disease [35, 36].

\subsubsection{Andrographolide}

Andrographolide, a labdane diterpenoid, is extracted from the rhizomes of andrographis paniculata. Andrographolide has been reported to reduce cellular lipid accumulation through its suppression of the SREBP pathway in vitro [37]. Recent evidence indicates that andrographolide inhibits lipogenic gene expression. Chen et al found that andrographolide attenuates FASN and SCD gene expression, which contributes to reduced lipid accumulation. Concomitantly, it represses C/EBP $\beta$ expression and activation by blocking the dual phosphorylation of CREB on Thr188 by ERK1/2 and on Ser184/Thr179 by glycogen synthase kinase (GSK)-3 $\beta$, leading to reduced adipogenesis in 3T3-L1 cells[38]. Moreover, andrographolide exhibits inhibitory effects on 3T3-L1 cell differentiation into mature adipocytes through its inhibition of the PPARY pathway[39]. Andrographolide can ameliorate lipid metabolism, which provides a promising approach for the treatment of obesity and other metabolic diseases.

Andrographolide exerts anticancer activities in vitro and in vivo. It inhibits the proliferation of numerous cancer cell lines, including breast cancer, lung cancer and melanoma cells[40]. Andrographolide suppresses MV4-11 cell proliferation by down-regulating FASN and ACC expression and up-regulating stromal interaction molecule 1 (STIM1) to block FA synthesis [41]. Therefore, andrographolide might retard metabolic disease through its inhibition of critical anabolic enzymes, such as FASN, ACC, and SCD, as well as transcription factors, including CREB, C/EBP $\beta$, and PPARY to impede lipogenesis.

\subsubsection{Shizukaol D}

Shizukaol D is a sesquiterpenoid isolated from chloranthus japonicas and was shown to activate AMPK and decrease triglyceride and cholesterol levels [42]. AMPK activation results in both the phosphorylation and inactivation of ACC and also the phosphorylation of MCD, leading to reduced malonyl-CoA levels and FA synthesis. Moreover, decreased malonyl-CoA promotes transportation of long-chain FAs into mitochondria mediated by CPT-1, resulting in increased FAO [43]. AMPK also down-regulates SREBP1c, which weakens the expression of enzymes involved in lipogenesis, including FASN and SCD-1, leading to decreased levels of monounsaturated FAs. Activation of AMPK boosts gene expression of PPAR $a$ and PPARr coactivator-1 alpha (PGC-1a), leading to augmentation of FAO [44]. Thus, shizukaol D plays a substantial role in inhibiting lipid accumulation through an AMPK-dependent mechanism and might be developed as a promising candidate for the treatment of metabolic diseases.

\subsubsection{Ginsenoside Rbl}

Ginsenoside $\mathrm{Rb} 1$, a tetracyclic triterpenoid compound isolated from ginseng, which is a traditional herbal medicine, exhibits activity against obesity and improves insulin sensitivity in vivo [45, 46].

Perilipin, a protein located on the surfaces of adipocyte lipid droplets, plays an important role in the regulation of lipolysis [47]. This protein inhibits tumor necrosis factor-a (TNF-a)-induced lipolysis in adipocytes, which causes decreased levels of free FAs (FFAs) and glycerine available for triglyceride synthesis in liver. Ginsenoside Rb1 could alleviate hepatic fat accumulation and suppress adipocyte lipolysis by up-regulating perilipin expression in adipocytes[48]. Park et al found that ginsenoside Rb1 decreases triglyceride and cholesterol levels that might occur through the down-regulation of cytochrome P-450 monooxygenase activity [49] and elevation of cAMP formation [50]. cAMP activates cAMP-dependent protein kinase A (PKA), which promotes phosphorylation and activation of HSL. Therefore, ginsenoside Rb1 effectively inhibits fat accumulation through the blockade of adipocyte 
lipolysis and might be used for treatment of NAFLD and atherosclerosis[51].

\subsection{Flavonoids and lipid metabolism}

Flavonoids are found in many plants and play an important role in the growth and development of plants as well as disease prevention. Flavonoids are bound to glycosides or exist in a free aglycone form. Flavonoids can be divided into several categories, including flavones, flavonols, flavanones, isoflavones, isoflavanones, chalcones, aurones, flavanols, and anthocyanidins[52]. Flavonoids have been shown to ameliorate lipid metabolism and can be used for prevention and treatment of metabolic disease (Table 2).

Citrus fruits such as oranges, grapefruits, bergamots and tangerines are good sources of citrus flavonoids [53]. Citrus flavonoids such as naringenin, hesperetin, eriodictyol, nobiletin and tangeretin are involved in the regulation of lipid metabolism and inhibition of hepatic apoB secretion[54, 55]. They are associated with decreased blood cholesterol and triglycerides in humans [56], and are capable of attenuating hepatic steatosis [57].

\subsubsection{Nobiletin}

Nobiletin, a polymethoxylated flavone, is separated from the peels of citrus depressa. Nobiletin administration promotes lipid catabolism and attenuates lipid accumulation [58]. Nobiletin enhances phosphorylation of HSL and ACC, which results in activation of HSL and inactivation of ACC.

The protein peroxisomal acyl-coenzyme A oxidase 1 (ACOX1) is a mitochondrial protein involved in peroxisomal FA catabolism and FAO [59]. Nobiletin also increases CPT-1 and ACOX1 expression and enhances $\beta$-oxidation of FAs through AMPK activation[60]. Nobiletin is capable of accelerating lipid catabolism in adipose tissue and might be considered as a natural candidate for the prevention and treatment of obesity [61].

\subsubsection{Tangeretin}

Some reports document the involvement of tangeretin in the regulation of lipid metabolism. Tangeretin can activate PPARa, but has no effect on intracellular lipid accumulation in 3T3L1 adipocytes [62]. Tangeretin might lead to decreased cellular accumulation of TAGs, which is the result of increased $\beta$-oxidation[63].

\subsubsection{Naringenin}

Naringenin, a flavanone in grapefruit, improves lipid metabolism in model creatures including zebra fish larvae[64] and rats[65]. Naringenin substantially increases PPARa, CPT-1 and uncoupling protein 2
(UCP-2) expression in rat liver [66] as well as hepatic FAO through the up-regulation of peroxisomal enzymes in mice[67]. Naringenin activates PPARa and PPARY ligand-binding domains, leading to increased FAO and reduced FA and cholesterol synthesis[68]. In addition, naringenin was reported to reduce hepatic SREBP-1c, resulting in decreased hepatic lipogenesis under fasting conditions [69].

Interestingly, naringenin could suppress 3-hydroxy-3-methylglutaryl-CoA reductase (HMGR), which is the rate-limiting enzyme in cholesterol synthesis, leading to reduced mevalonic acid and cholesterol production[70]. Overall, naringenin exerts both hypolipidemic and anti-adiposity effects and might protect against metabolic diseases.

\subsubsection{Hesperetin}

Hesperetin, the main ingredient of the extract from S. edule shoots, can activate PPARa and PPARY impacting lipid metabolism. It was reported to suppress oleic acid-induced lipid accumulation in HepG2 cells and reduce hepatic lipogenesis. In a high-fat-diet-induced animal model, in combination with caffeic acid, hesperetin treatment attenuated adipose tissue fat and prevented a fatty liver by up-regulation of AMPK to decrease expression of lipogenic enzymes and stimulate lipolysis [71].

\subsubsection{Luteolin}

Luteolin ( $3^{\prime}, 4^{\prime}, 5,7$-tetrahydroxyflavone), another member of the flavonoid family, is a natural compound derived from plants that shows anti-lipogenic functions. Luteolin reduces lipid levels by promoting FAO and inhibiting FA synthesis. Brusselmans et al reported that luteolin reduces lipogenesis by inhibiting FASN activity in prostate and breast cancer cells, which was associated with its capability to arrest growth and induce apoptosis[72]. Liu et al showed that luteolin exerts lipid-lowering effects in HepG2 cells, which might be due to the up-regulation of CPT-1 and down-regulation of SREBP-1c and FASN. This might be attributed to the phosphorylation and activation of AMPKa and inactivation of ACC[73].

Luteolin also exerts inhibitory effects on phospholipid and cholesterol synthesis[72, 74]. Luteolin inhibits ceramide anabolism to generate complex sphingolipids, including both sphingomyelin and glycosphingolipid, in colon cancer cells[74]. This compound has been recently reported to suppress lung cancer in Swiss albino mice [75]. Lim et al found that luteolin reduces cholesterol synthesis by inhibiting the level of certain enzymes, such as HMG-CoA synthase 1 (HMGCS1), mevalonate kinase (MVK), or squalene epoxidase 
(SQLE) in choriocarcinoma cell lines [76]. Thus, luteolin treatment might have benefits for cancer prevention and therapy by blocking lipid anabolic processes.

\subsubsection{Quercetin}

Quercetin is widely found in fruits and vegetables in the human diet. This compound acts as a potent inhibitor of lipogenesis in prostate and breast cancer cells through its inhibition of FASN activity [72]. It also inhibits lipid synthesis by suppressing
PPARy and C/EBPa activities and activating AMPK in 3T3-L1 cells[77].

Moreover, quercetin has been shown to regulate hepatic cholesterol metabolism. It significantly increased the activity of hepatic cholesterol 7a-hydroxylase, an enzyme involved in cholesterol metabolism, promoting cholesterol-to-bile acid conversion [78]. Quercetin also elevates the expression of liver $X$ receptor a $(\mathrm{LXRa})$ that is associated with the accelerated conversion of cholesterol to bile acids.

Table 2. Structure of flavonoids and their mechanisms of action targeting lipid metabolism

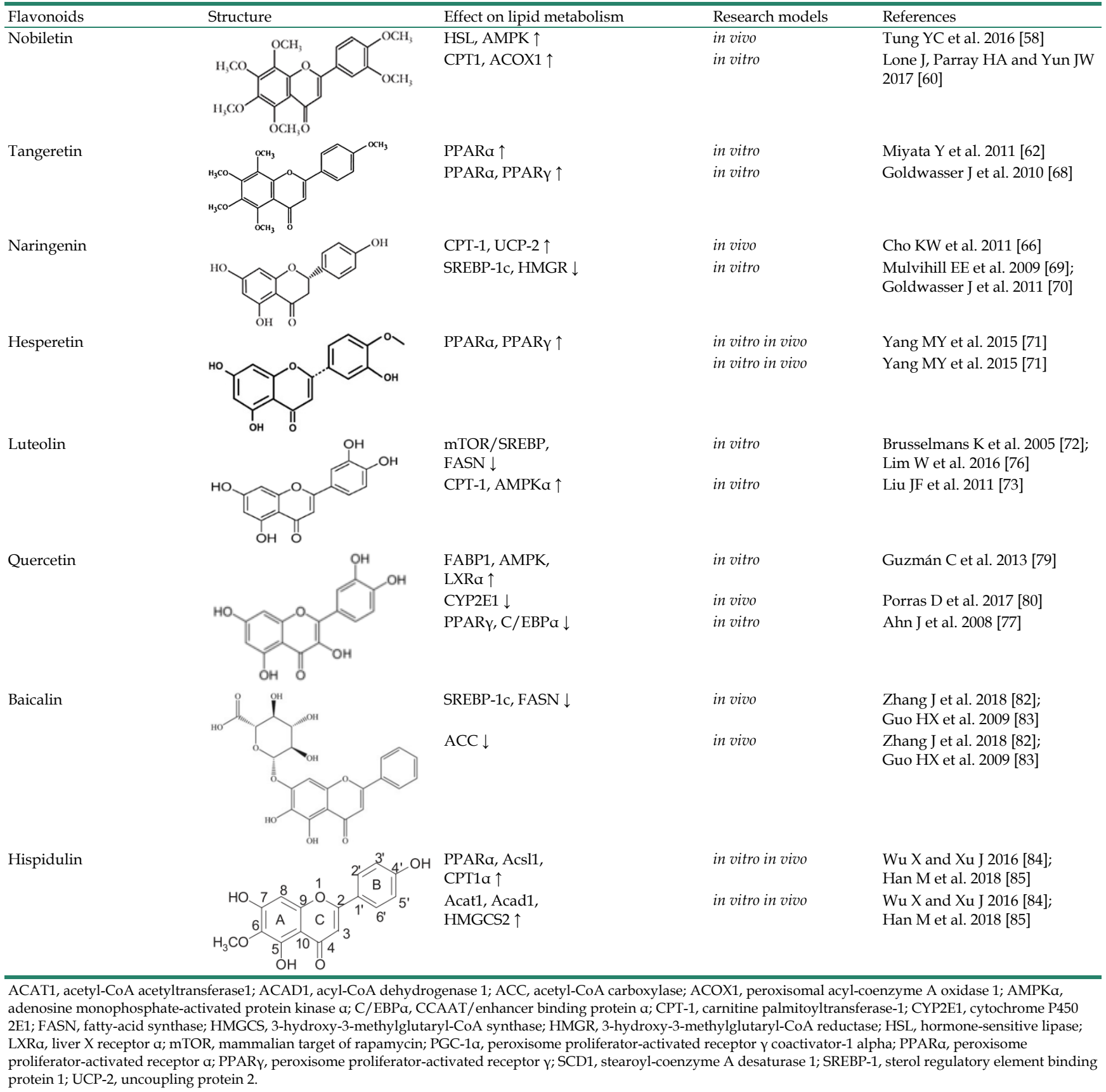


The expression of FA binding protein 1 (FABP1) is known to be decreased in patients with NAFLD. FABP1 repression is related to the down-regulation of forkhead box protein A1 (FOXA1) and increased $\mathrm{C} / \mathrm{EBPa}$, resulting in excessive lipid accumulation [79]. Quercetin attenuates FABP1 downregulation and ameliorates hepatic steatosis. In addition, quercetin suppresses excessive cytochrome P450 2E1(CYP2E1) overexpression to attenuate the development of NAFLD [80].

Hence, quercetin might reduce lipid accumulation and have good therapeutic potential against NAFLD and cancer [81].

\subsubsection{Baicalin}

Baicalin is a flavonoid that is extracted from the root of scutellaria baicalensis georgi. It assists in the attenuation of steatohepatitis by suppressing crucial regulators of lipid metabolism.

Several studies demonstrated that baicalin inhibits lipid synthesis by down-regulating SREBP-1c and FASN and enhancing FAO by stimulating phosphorylation of ACC in vivo[82, 83].

\subsubsection{Hispidulin}

Hispidulin(4',5,7-trihydroxy-6-methoxyflavone) is a flavonoid extracted from saussurea involucrate, a traditional Chinese herbal medicine. Hispidulin exerts hypolipidemic effects and has therapeutic potential against dyslipidemia[84]. Wu et al reported that hispidulin acts as a PPARa agonist and increases the expression of genes involved in FAO, such as longchain acyl-CoA synthetase 1(Acsl 1), CPT1a, acetyl-CoA acetyltransferase1 (Acat1), acyl-CoA dehydrogenase 1 (Acad1) and HMG-CoA synthase 2 (HMGCS2).

In addition, hispidulin inhibits hepatocellular carcinoma cell growth and metastasis through the activation of PPARy, which was associated with increased phosphorylation of AMPK and ERK1/2 [85]. Thus, besides its hypolipidemic function, hispidulin might also contribute to cancer therapy by targeting FAO.

\section{Conclusions}

The balance of lipid anabolic and catabolic metabolism is fine-tuned to optimize the metabolic requirements in organisms. During the pathogenic development of metabolic diseases with lipid metabolic disorders such as NAFLD, obesity, and cancer, the lipogenic/lipolytic balance is interrupted, which results in over-activated lipogenesis and excessive lipid accumulation.

A vast number of natural compounds are being studied for the management of lipid metabolic-related diseases. Treatment with natural compounds described in this review has been demonstrated in cell-based assays, animal-based assays, and even clinical studies, to delay and ameliorate the development of disease by regulating lipid metabolism. From the perspective of mechanism of action, despite the target diversity and specificity for each species of natural compound, the molecular target is mainly focused on nodal metabolic enzymes that control lipid transportation, conversion and utilization, including CPT-1, FASN, and ACC. Another class of targets is transcription factors as well as co-regulators, such as SREBP-1, PPARa, PPAR $\gamma$, and $\mathrm{C} / \mathrm{EBPa}$ that initiate lipid metabolic programs. From the perspective of clinical application, although the associated evidence is limited, most of these natural compounds have been demonstrated to attenuate metabolic-associated diseases without severe toxicity or health hazard concerns. Nevertheless, due to the complex signaling circuits integrated compensatory redundancies account for the progression of global metabolic disorders, making recapitulating the scenario in vitro difficult. Clinical investigations and appropriately designed studies are crucial to elucidate the true influence of these compounds in patients with lipid metabolic-related diseases.

\section{Acknowledgements}

This work was supported by grants from the National Natural Science Foundation of China (81874195, 81573014), the Natural Science Foundation of Hunan Province (2016JJ2171).

\section{Competing Interests}

The authors have declared that no competing interest exists.

\section{References}

1. C Wang, J Ma, N Zhang, et al. The acetyl-CoA carboxylase enzyme: a target for cancer therapy? Expert Rev Anticancer Ther. 2015; 15(6): 667-76.

2. C R Santos and A Schulze. Lipid metabolism in cancer. FEBS J. 2012; 279(15): 2610-23.

3. M J Watt and Y Cheng. Triglyceride metabolism in exercising muscle. Biochim Biophys Acta. 2017; 1862(10 Pt B): 1250-9.

4. $\mathrm{H} \mathrm{He}, \mathrm{H} \mathrm{H}$ Liu, J W Wang, et al. Molecular cloning of the goose ACSL3 and ACSL5 coding domain sequences and their expression characteristics during goose fatty liver development. Mol Biol Rep. 2014; 41(4): 2045-53.

5. N Casals, V Zammit, L Herrero, et al. Carnitine palmitoyltransferase 1C: From cognition to cancer. Prog Lipid Res. 2016; 61: 134-48.

6. $\mathrm{R} \mathrm{Ke}, \mathrm{Q} \mathrm{Xu}, \mathrm{C} \mathrm{Li}$, et al. Mechanisms of AMPK in the maintenance of ATP balance during energy metabolism. Cell Biol Int. 2018; 42(4): 384-92.

7. L Luo, S Jiang, D Huang, et al. MLK3 phophorylates AMPK independently of LKB1. PLoS One. 2015; 10(4): e0123927.

8. M Pawlak, P Lefebvre, and B Staels. Molecular mechanism of PPARalpha action and its impact on lipid metabolism, inflammation and fibrosis in non-alcoholic fatty liver disease. J Hepatol. 2015; 62(3): 720-33.

9. E Bedu, W Wahli, and B Desvergne. Peroxisome proliferator-activated receptor beta/delta as a therapeutic target for metabolic diseases. Expert Opin Ther Targets. 2005; 9(4): 861-73.

10. J V Jester, E Potma, and D J Brown. PPARgamma Regulates Mouse Meibocyte Differentiation and Lipid Synthesis. Ocul Surf. 2016; 14(4): 484-94. 
11. Y Zhou, Y L Ding, J L Zhang, et al. Alpinetin improved high fat diet-induced non-alcoholic fatty liver disease (NAFLD) through improving oxidative stress, inflammatory response and lipid metabolism. Biomed Pharmacother. 2018; 97: 1397-408.

12. M Vacca, M Allison, J L Griffin, et al. Fatty Acid and Glucose Sensors in Hepatic Lipid Metabolism: Implications in NAFLD. Semin Liver Dis. 2015; 35(3): 250-61.

13. G Musso, R Gambino, and M Cassader. Recent insights into hepatic lipid metabolism in non-alcoholic fatty liver disease (NAFLD). Prog Lipid Res. 2009; 48(1): $1-26$

14. J Magdalon and W T Festuccia. Regulation of adiposity by mTORC1. Einstein (Sao Paulo). 2017; 15(4): 507-11.

15. Z Tan, L Xiao, M Tang, et al. Targeting CPT1A-mediated fatty acid oxidation sensitizes nasopharyngeal carcinoma to radiation therapy. Theranostics. 2018; 8(9): 2329-47.

16. X Luo, L Hong, C Cheng, et al. DNMT1 mediates metabolic reprogramming induced by Epstein-Barr virus latent membrane protein 1 and reversed by grifolin in nasopharyngeal carcinoma. Cell Death Dis. 2018; 9(6): 619.

17. X Luo, $\mathrm{C}$ Cheng, $\mathrm{Z}$ Tan, et al. Emerging roles of lipid metabolism in cancer metastasis. Mol Cancer. 2017; 16(1): 76.

18. X Luo, $\mathrm{X}$ Zhao, $\mathrm{C}$ Cheng, et al. The implications of signaling lipids in cancer metastasis. Exp Mol Med. 2018; 50(9): 127.

19. L Xiao, Z Y Hu, X Dong, et al. Targeting Epstein-Barr virus oncoprotein LMP1-mediated glycolysis sensitizes nasopharyngeal carcinoma to radiation therapy. Oncogene. 2014; 33(37): 4568-78.

20. J Long, C J Zhang, N Zhu, et al. Lipid metabolism and carcinogenesis, cancer development. Am J Cancer Res. 2018; 8(5): 778-91.

21. R C Alves, R P Fernandes, J O Eloy, et al. Characteristics, Properties and Analytical Methods of Paclitaxel: A Review. Crit Rev Anal Chem. 2018; 48(2): $110-8$.

22. C Dziggel, H Schafer, and M Wink. Tools of pathway reconstruction and production of economically relevant plant secondary metabolites in recombinant microorganisms. Biotechnol J. 2017; 12(1).

23. T Xu, Q Pang, Y Wang, et al. Betulinic acid induces apoptosis by regulating PI3K/Akt signaling and mitochondrial pathways in human cervical cancer cells. Int J Mol Med. 2017; 40(6): 1669-78.

24. L Potze, S Di Franco, C Grandela, et al. Betulinic acid induces a novel cell death pathway that depends on cardiolipin modification. Oncogene. 2016; 35(4): 427-37.

25. A E Abd El-Wahab, D A Ghareeb, E E Sarhan, et al. In vitro biological assessment of Berberis vulgaris and its active constituent, berberine: antioxidants, anti-acetylcholinesterase, anti-diabetic and anticancer effects. BMC Complement Altern Med. 2013; 13: 218.

26. J Peng, Y C Lv, P P He, et al. Betulinic acid downregulates expression of oxidative stress-induced lipoprotein lipase via the PKC/ERK/c-Fos pathway in RAW264.7 macrophages. Biochimie. 2015; 119: 192-203.

27. H Yan, D X Zhang, X Shi, et al. Activation of the prolyl-hydroxylase oxygen-sensing signal cascade leads to AMPK activation in cardiomyocytes. J Cell Mol Med. 2012; 16(9): 2049-59.

28. H Y Quan, D Y Kim, S J Kim, et al. Betulinic acid alleviates non-alcoholic fatty liver by inhibiting SREBP1 activity via the AMPK-mTOR-SREBP signaling pathway. Biochem Pharmacol. 2013; 85(9): 1330-40.

29. E Martinez-Abundis, M Mendez-Del Villar, K G Perez-Rubio, et al. Novel nutraceutic therapies for the treatment of metabolic syndrome. World J Diabetes. 2016; 7(7): 142-52.

30. W L Zhang, L Zhu, and J G Jiang. Active ingredients from natural botanicals in the treatment of obesity. Obes Rev. 2014; 15(12): 957-67.

31. Y Li, Z Kang, S Li, et al. Ursolic acid stimulates lipolysis in primary-cultured rat adipocytes. Mol Nutr Food Res. 2010; 54(11): 1609-17.

32. Y Jia, M J Bhuiyan, H J Jun, et al. Ursolic acid is a PPAR-alpha agonist that regulates hepatic lipid metabolism. Bioorg Med Chem Lett. 2011; 21(19): 5876-80.

33. Q-y Zheng, F-s Jin, C Yao, et al. Ursolic acid-induced AMP-activated protein kinase (AMPK) activation contributes to growth inhibition and apoptosis in human bladder cancer T24 cells. Biochemical and Biophysical Research Communications. 2012; 419(4): 741-7.

34. A Lodi, A Saha, X Lu, et al. Combinatorial treatment with natural compounds in prostate cancer inhibits prostate tumor growth and leads to key modulations of cancer cell metabolism. NPJ Precis Oncol. 2017; 1.

35. J S Li, W J Wang, Y Sun, et al. Ursolic acid inhibits the development of nonalcoholic fatty liver disease by attenuating endoplasmic reticulum stress. Food Funct. 2015; 6(5): 1643-51.

36. S Li, X Liao, F Meng, et al. Therapeutic role of ursolic acid on ameliorating hepatic steatosis and improving metabolic disorders in high-fat diet-induced non-alcoholic fatty liver disease rats. PLoS One. 2014; 9(1): e86724.

37. L Ding, J Li, B Song, et al. Andrographolide prevents high-fat diet-induced obesity in C57BL/ 6 mice by suppressing the sterol regulatory element-binding protein pathway. J Pharmacol Exp Ther. 2014; 351(2): 474-83.

38. C C Chen, W T Chuang, A H Lin, et al. Andrographolide inhibits adipogenesis of 3T3-L1 cells by suppressing C/EBPbeta expression and activation. Toxicol Appl Pharmacol. 2016; 307: 115-22.

39. L Jin, W Fang, B Li, et al. Inhibitory effect of andrographolide in 3T3-L1 adipocytes differentiation through the PPARgamma pathway. Mol Cell Endocrinol. 2012; 358(1): 81-7.
40. J C Lim, T K Chan, D S Ng, et al. Andrographolide and its analogues: versatile bioactive molecules for combating inflammation and cancer. Clin Exp Pharmacol Physiol. 2012; 39(3): 300-10.

41. X Chen, J Zhang, L Yuan, et al. Andrographolide Suppresses MV4-11 Cell Proliferation through the Inhibition of FLT3 Signaling, Fatty Acid Synthesis and Cellular Iron Uptake. Molecules. 2017; 22(9).

42. $\mathrm{R} \mathrm{Hu}, \mathrm{H}$ Yan, $\mathrm{X}$ Hao, et al. Shizukaol D isolated from Chloranthus japonicas inhibits AMPK-dependent lipid content in hepatic cells by inducing mitochondrial dysfunction. PLoS One. 2013; 8(8): e73527.

43. V A Zammit and A Arduini. The AMPK-malonyl-CoA-CPT1 axis in the control of hypothalamic neuronal function. Cell Metab. 2008; 8(3): 175

44. R B Vega, J M Huss, and D P Kelly. The coactivator PGC-1 cooperates with peroxisome proliferator-activated receptor alpha in transcriptional control of nuclear genes encoding mitochondrial fatty acid oxidation enzymes. Mol Cell Biol. 2000; 20(5): 1868-76.

45. L Shen, Y Xiong, D Q Wang, et al. Ginsenoside Rb1 reduces fatty liver by activating AMP-activated protein kinase in obese rats. J Lipid Res. 2013; 54(5): $1430-8$

46. Y Xiong, L Shen, K J Liu, et al. Antiobesity and antihyperglycemic effects of ginsenoside Rb1 in rats. Diabetes. 2010; 59(10): 2505-12.

47. D L Brasaemle. Thematic review series: adipocyte biology. The perilipin family of structural lipid droplet proteins: stabilization of lipid droplets and control of lipolysis. J Lipid Res. 2007; 48(12): 2547-59.

48. X Yu, L Ye, H Zhang, et al. Ginsenoside Rb1 ameliorates liver fat accumulation by upregulating perilipin expression in adipose tissue of $\mathrm{db} / \mathrm{db}$ obese mice. J Ginseng Res. 2015; 39(3): 199-205.

49. A M Jenner and J A Timbrell. Influence of inducers and inhibitors of cytochrome P450 on the hepatotoxicity of hydrazine in vivo. Arch Toxicol. 1994; 68(6): 349-57

50. K H Park, H J Shin, Y B Song, et al. Possible role of ginsenoside Rb1 on regulation of rat liver triglycerides. Biol Pharm Bull. 2002; 25(4): 457-60.

51. L Qiao, X Zhang, M Liu, et al. Ginsenoside Rb1 Enhances Atherosclerotic Plaque Stability by Improving Autophagy and Lipid Metabolism in Macrophage Foam Cells. Front Pharmacol. 2017; 8: 727.

52. E E Mulvihill and M W Huff. Antiatherogenic properties of flavonoids: implications for cardiovascular health. Can J Cardiol. 2010; 26 Suppl A: 17A-21A.

53. E Tripoli, M La Guardia, S Giammanco, et al. Citrus flavonoids: Molecular structure, biological activity and nutritional properties: A review. Food Chemistry. 2007; 104(2): 466-79.

54. Y Lin, M A Vermeer, W Bos, et al. Molecular structures of citrus flavonoids determine their effects on lipid metabolism in HepG2 cells by primarily suppressing apoB secretion. J Agric Food Chem. 2011; 59(9): 4496-503.

55. N M Borradaile, L E de Dreu, and M W Huff. Inhibition of net HepG2 cell apolipoprotein B secretion by the citrus flavonoid naringenin involves activation of phosphatidylinositol 3-kinase, independent of insulin receptor substrate-1 phosphorylation. Diabetes. 2003; 52(10): 2554-61.

56. C E Chandler, D E Wilder, J L Pettini, et al. CP-346086: an MTP inhibitor that lowers plasma cholesterol and triglycerides in experimental animals and in humans. J Lipid Res. 2003; 44(10): 1887-901.

57. E E Mulvihill, A C Burke, and M W Huff. Citrus Flavonoids as Regulators of Lipoprotein Metabolism and Atherosclerosis. Annu Rev Nutr. 2016; 36: 275-99.

58. Y C Tung, S Li, Q Huang, et al. 5-Demethylnobiletin and 5-Acetoxy-6,7,8,3',4'-pentamethoxyflavone Suppress Lipid Accumulation by Activating the LKB1-AMPK Pathway in 3T3-L1 Preadipocytes and High Fat Diet-Fed C57BL/6 Mice. J Agric Food Chem. 2016; 64(16): 3196-205.

59. G Yan, X Li, Y Peng, et al. The Fatty Acid beta-Oxidation Pathway is Activated by Leucine Deprivation in HepG2 Cells: A Comparative Proteomics Study. Sci Rep. 2017; 7(1): 1914

60. J Lone, H A Parray, and J W Yun. Nobiletin induces brown adipocyte-like phenotype and ameliorates stress in 3T3-L1 adipocytes. Biochimie.2018;146:97-104.

61. Y S Lee, B Y Cha, S S Choi, et al. Nobiletin improves obesity and insulin resistance in high-fat diet-induced obese mice. J Nutr Biochem. 2013; 24(1): $156-62$.

62. Y Miyata, H Tanaka, A Shimada, et al. Regulation of adipocytokine secretion and adipocyte hypertrophy by polymethoxyflavonoids, nobiletin and tangeretin. Life Sci. 2011; 88(13-14): 613-8.

63. E M Kurowska, J A Manthey, A Casaschi, et al. Modulation of HepG2 cell net apolipoprotein B secretion by the citrus polymethoxyflavone, tangeretin. Lipids. 2004; 39(2): 143-51.

64. H Lin, Z Zhou, W Zhong, et al. Naringenin inhibits alcoholic injury by improving lipid metabolism and reducing apoptosis in zebrafish larvae. Oncol Rep. 2017; 38(5): 2877-84.

65. B Ren, W Qin, F Wu, et al. Apigenin and naringenin regulate glucose and lipid metabolism, and ameliorate vascular dysfunction in type 2 diabetic rats. Eur J Pharmacol. 2016; 773: 13-23.

66. K W Cho, Y O Kim, J E Andrade, et al. Dietary naringenin increases hepatic peroxisome proliferators-activated receptor alpha protein expression and decreases plasma triglyceride and adiposity in rats. Eur J Nutr. 2011; 50(2): 81-8.

67. D T Huong, Y Takahashi, and T Ide. Activity and mRNA levels of enzymes involved in hepatic fatty acid oxidation in mice fed citrus flavonoids. Nutrition. 2006; 22(5): 546-52. 
68. J Goldwasser, P Y Cohen, E Yang, et al. Transcriptional regulation of human and rat hepatic lipid metabolism by the grapefruit flavonoid naringenin: role of PPARalpha, PPARgamma and LXRalpha. PLoS One. 2010; 5(8): e12399.

69. E E Mulvihill, E M Allister, B G Sutherland, et al. Naringenin prevents dyslipidemia, apolipoprotein B overproduction, and hyperinsulinemia in LDL receptor-null mice with diet-induced insulin resistance. Diabetes. 2009; 58(10): 2198-210.

70. J Goldwasser, P Y Cohen, W Lin, et al. Naringenin inhibits the assembly and long-term production of infectious hepatitis $C$ virus particles through a PPAR-mediated mechanism. J Hepatol. 2011; 55(5): 963-71.

71. M Y Yang, K C Chan, Y J Lee, et al. Sechium edule Shoot Extracts and Active Components Improve Obesity and a Fatty Liver That Involved Reducing Hepatic Lipogenesis and Adipogenesis in High-Fat-Diet-Fed Rats. J Agric Food Chem. 2015; 63(18): 4587-96.

72. K Brusselmans, R Vrolix, G Verhoeven, et al. Induction of cancer cell apoptosis by flavonoids is associated with their ability to inhibit fatty acid synthase activity. J Biol Chem. 2005; 280(7): 5636-45.

73. J F Liu, Y Ma, Y Wang, et al. Reduction of lipid accumulation in HepG2 cells by luteolin is associated with activation of AMPK and mitigation of oxidative stress. Phytother Res. 2011; 25(4): 588-96.

74. L Abdel Hadi, C Di Vito, G Marfia, et al. Sphingosine Kinase 2 and Ceramide Transport as Key Targets of the Natural Flavonoid Luteolin to Induce Apoptosis in Colon Cancer Cells. PLoS One. 2015; 10(11): e0143384.

75. E R Kasala, L N Bodduluru, C C Barua, et al. Antioxidant and antitumor efficacy of Luteolin, a dietary flavone on benzo(a)pyrene-induced experimental lung carcinogenesis. Biomed Pharmacother. 2016; 82: 568-77.

76. W Lim, C Yang, F W Bazer, et al. Luteolin Inhibits Proliferation and Induces Apoptosis of Human Placental Choriocarcinoma Cells by Blocking the PI3K/AKT Pathway and Regulating Sterol Regulatory Element Binding Protein Activity. Biol Reprod. 2016; 95(4): 82.

77. J Ahn, H Lee, S Kim, et al. The anti-obesity effect of quercetin is mediated by the AMPK and MAPK signaling pathways. Biochem Biophys Res Commun. 2008; 373(4): 545-9.

78. M Zhang, Z Xie, W Gao, et al. Quercetin regulates hepatic cholesterol metabolism by promoting cholesterol-to-bile acid conversion and cholesterol efflux in rats. Nutr Res. 2016; 36(3): 271-9.

79. C Guzman, M Benet, S Pisonero-Vaquero, et al. The human liver fatty acid binding protein (FABP1) gene is activated by FOXA1 and PPARalpha; and repressed by C/EBPalpha: Implications in FABP1 down-regulation in nonalcoholic fatty liver disease. Biochim Biophys Acta. 2013; 1831(4): 803-18.

80. D Porras, E Nistal, S Martinez-Florez, et al. Protective effect of quercetin on high-fat diet-induced non-alcoholic fatty liver disease in mice is mediated by modulating intestinal microbiota imbalance and related gut-liver axis activation. Free Radic Biol Med. 2017; 102: 188-202.

81. A K Stoldt, M Mielenz, G Nurnberg, et al. Effects of a six-week intraduodenal supplementation with quercetin on liver lipid metabolism and oxidative stress in peripartal dairy cows. J Anim Sci. 2016; 94(5): 1913-23.

82. J Zhang, $\mathrm{H}$ Zhang, $\mathrm{X}$ Deng, et al. Baicalin attenuates non-alcoholic steatohepatitis by suppressing key regulators of lipid metabolism, inflammation and fibrosis in mice. Life Sci. 2018; 192: 46-54.

83. H X Guo, D H Liu, Y Ma, et al. Long-term baicalin administration ameliorates metabolic disorders and hepatic steatosis in rats given a high-fat diet. Acta Pharmacol Sin. 2009; 30(11): 1505-12.

84. X Wu and J Xu. New Role of Hispidulin in Lipid Metabolism: PPARalpha Activator. Lipids. 2016; 51(11): 1249-57.

85. M Han, H Gao, P Ju, et al. Hispidulin inhibits hepatocellular carcinoma growth and metastasis through AMPK and ERK signaling mediated activation of PPARgamma. Biomed Pharmacother. 2018; 103: 272-83. 